\title{
Surrogate End Points, Health Outcomes, and the Drug-Approval Process for the Treatment of Risk Factors for Cardiovascular Disease
}

Bruce M. Psaty, MD, PhD

Noel S. Weiss, MD, DrPH

Curt D. Furberg, MD, PhD

Thomas D. Koepsell, MD, MPH

David S. Siscovick, MD, MPH

Frits R. Rosendaal, MD, PhD

Nicholas L. Smith, PhD, MPH

Susan R. Heckbert, MD, PhD

Robert C. Kaplan, MS

Danyu Lin, PhD

Thomas R. Fleming, PhD

Edward H. Wagner, MD, MPH

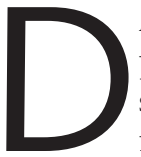

ATA ON SURROGATE END POINTS SUCH AS BLOOD pressure or body weight have often been used to support the approval of new pharmacologic treatments for cardiovascular risk factors. In small, short-term studies, a new drug reduces the level of a risk factor, and the changes in risk factor levels are interpreted as if the health benefits expected on the basis of those changes will necessarily follow. An editorial on the pharmacotherapy of obesity illustrates the $\operatorname{argument}^{1}$ : in the context of discussing the association between appetite suppressant drugs and primary pulmonary hypertension, ${ }^{2}$ the editorialists used observational evidence on the association of body mass index with mortality and translated data on weight loss in a small, short-term trial of dexfenfluramine $e^{3}$ into an estimate of lives that could be saved by long-term drug therapy for obesity. The US Food and Drug Administration (FDA) approved dexfenfluramine on the basis of this same surrogate end point argument $t^{4}$ : "the potential health benefits of anorectic drugs outweigh their risk when considered against the health hazards of obesity." "When, after the drug was approved, the adverse effects were found to be greater than estimated on the basis of preapproval trials, ${ }^{6,7}$ the drug was withdrawn. Is this an example of the drug-approval pro-

\section{See also p 790.}

cess working well, or does it point to a fundamental flaw in the way drugs are approved?

Surrogate end points sometimes fail to serve as valid predictors of important health outcomes. ${ }^{8}$ One remedy would be to require, prior to approving new drug therapies for cardiovascular risk factors, large, long-term clinical trials to assess the drug's effects on major disease end points. The historical precedent of having accepted surrogate end points and the current interest in minimizing the time to drug approval may make this approach impracticable. Alternatively, a regular requirement for phase 4 trials would perhaps be a practical and achievable strategy for drug approval, one that improves incrementally upon the current approach, which usually requires no other evaluation than the use of surrogate end points.

\section{Cardiovascular Risk Factors as Surrogate End Points}

Surrogate end points traditionally have been used to approve drug therapies for cardiovascular risk factors-obesity, hypertension, hypercholesterolemia, and diabetes mellitus. An argument based on surrogates appears to have the logical appeal and the formal structure of deduction: a risk factor causes morbidity and mortality, and the intervention reduces the risk factor level; therefore, the intervention will reduce the risk of morbidity and mortality. ${ }^{9}$ This rationale represents an argument by analogy rather than empirical evidence.

Many cardiovascular risk factors present as asymptomatic conditions. With rare exceptions, hypertension and hypercholesterolemia do not produce symptoms before a cardiovascular event. The symptoms of type 2 diabetes mellitus are often so mild that estimating the date of onset is difficult. These risk fac-

Author Affiliations: The Cardiovascular Health Research Unit, the Departments of Medicine (Drs Psaty, Koepsell, Siscovick, and Smith), Epidemiology (Drs Psaty, Weiss, Koepsell, Siscovick, and Heckbert, and Mr Kaplan), Health Services (Drs Psaty, Koepsell, and Wagner), and Biostatistics (Drs Lin and Fleming), University of Washington, Seattle; the Department of Public Health Sciences, Wake Forest University School of Medicine, Winston-Salem, NC (Dr Furberg); the Department of Clinical Epidemiology, Leiden University Medical Center, Leiden, the Netherlands (Dr Rosendaal); and the Center for Health Studies, Group Health Cooperative of Puget Sound, Seattle (Dr Wagner).

Financial Disclosure: Dr Psaty serves on the events committee for a clinical trial sponsored by Wyeth-Ayerst. Dr Furberg has received research grants from Pfizer and WyethAyerst and served on data safety monitoring boards for Parke-Davis and Searle. Corresponding Author and Reprints: Bruce M. Psaty, MD, PhD, Cardiovascular Health Research Unit, Suite \#1360, 1730 Minor Ave, Seattle, WA 98101.

Controversies Section Editor: Phil B. Fontanarosa, MD, Interim Coeditor

C(1999 American Medical Association. All rights reserved. 
tors are associated with major complications, most commonly stroke, myocardial infarction, renal failure, and congestive heart failure. Although physicians routinely monitor risk factor levels during therapy, the purpose of treatment is to reduce the occurrence of these often devastating complications.

Drug therapies for these risk factors are often long-term, sometimes lifelong. For most patients, the absolute risk of cardiovascular disease is low. For instance, in the Systolic Hypertension in the Elderly Program ${ }^{10}$ the rates for the combined end points of stroke or coronary disease were 2.71 and 1.87 per 100 person-years in the placebo and treated groups, respectively. The risk difference of 0.84 events means that in order to prevent 1 cardiovascular event, about 120 older adults with isolated systolic hypertension need to be treated for 1 year. Most treated patients will receive little benefit. If there are unanticipated adverse effects, even relatively uncommon ones may minimize or eliminate the average health benefits expected from drug therapy.

Surrogate end points have several potential advantages. Clinical trials evaluating surrogate end points require smaller sample sizes, and they can sometimes be completed in weeks or months rather than years. If the end point is lipid levels, trials of lipid-lowering therapy typically include around 100 patients observed for 3 to 12 months. If the end point is the incidence of cardiovascular events, the trials often require several thousand patients observed for 4 to 5 years. ${ }^{11,12}$ The ability to bring potentially effective therapies to clinical practice quickly and inexpensively makes surrogate end points attractive in the drug-approval process.

The principal disadvantage of using surrogates to assess therapies is the possibility of an incomplete, inadequate, or misleading evaluation. ${ }^{8,13,14}$ To use only a surrogate end point is to accept as empirical evidence for clinical practice a hypothesis about health benefits that has never been tested. Drug therapies usually have multiple effects, and resorting to a single surrogate end point that focuses exclusively on 1 intermediate effect often precludes the evaluation of other intended or unintended health effects. While even large clinical trials may not be able to provide a complete evaluation of safety for rare adverse events, the small sample sizes and short trial durations typically required in surrogate end point trials provide little assurance of long-term safety.

\section{Clinical Trials of Drug Therapies for Cardiovascular Risk Factors}

The results of long-term trials of lipid level reduction have been mixed. In men with hypercholesterolemia, clofibrate was associated with a $20 \%$ decrease in the risk of ischemic heart disease but a $44 \%$ increase in the risk of total mortality. ${ }^{15,16}$ In a meta-analysis of cholesterol reduction trials, ${ }^{17}$ the fibrates were associated with a 30\% increase in the risk of noncoronary disease mortality (95\% confidence interval $[\mathrm{CI}], 8 \%-56 \%)$ and a $19 \%$ increase in total mortality (95\% CI, 3\%-33\%). In part as a result of the adverse effects seen with clofibrate, the FDA encouraged the pharmaceutical in-

(C)1999 American Medical Association. All rights reserved. dustry to mount long-term trials and evaluate 3-hydroxy3-methylglutaryl coenzyme A (HMG-CoA) reductase inhibitors in terms of major disease end points. The large trials evaluating simvastatin and pravastatin have recently demonstrated important reductions in the occurrence of cardiovascular events in patients with and without preexisting coronary disease. ${ }^{11,12,18}$ Thus, some important health outcomes of cholesterol-lowering trials appear to depend on the class of drug used to lower cholesterol level.

Similarly, evidence from large trials of hypertension treatments suggests that the findings for the surrogate end point of blood pressure reduction may fail to mirror those for the end point of incidence of coronary disease. In a meta-analysis, ${ }^{19}$ low-dose diuretic therapy was associated with a reduced risk of coronary heart disease (relative risk reduction [RRR], 28\%; 95\% CI, 15\%-39\%), but not for high-dose diuretic therapy (RRR, $1 \% ; 95 \% \mathrm{CI},-18 \%$ to $17 \%$ ) or $\beta$-blocker therapy (RRR, $7 \% ; 95 \%$ CI, $-9 \%$ to $20 \%$ ). While all 3 regimens lowered blood pressure, drug treatment with high-dose diuretic therapy or $\beta$-blocker therapy failed to prevent coronary heart disease. Some of the possible explanations for the lack of benefit of high-dose diuretics have been reviewed. ${ }^{20}$ The health outcomes of blood pressure reduction appear to depend not only on the type but perhaps also on the dose of the drug.

The largest US trial demonstrating the health effects of treating type 2 diabetes mellitus is still the University Group Diabetes Program. ${ }^{21}$ In this placebo-controlled trial, tolbutamide was associated with a doubling of the risk of cardiovascular mortality (relative risk [RR], 2.61; 95\% CI, 1.295.27). In contrast to the findings for type 1 diabetes mellitus, ${ }^{22}$ intensive treatment of type 2 diabetes mellitus, compared with usual care, has yielded equivocal results. While intensive treatment was associated with delayed onset of microvascular disease in one trial, ${ }^{23}$ the findings of another trial ${ }^{24}$ suggested that persons who receive intensive treatment for type 2 diabetes mellitus may have a higher cardiovascular mortality rate than those who receive usual care (RR, 1.67; 95\% CI, 0.88-3.13). In a recent UK trial of patients with type 2 diabetes, intensive blood glucose control with insulin or sulfonylureas was associated with a decreased risk of microvascular but not macrovascular complications. ${ }^{25}$ However, the interpretation of this large, complex trial is difficult. ${ }^{26}$

The effects of drug therapies on levels of glucose and glycosylated hemoglobin or even their effects on the occurrence and progression of microvascular disease may not predict their overall health effects on macrovascular disease. ${ }^{27}$ In patients with type 2 diabetes mellitus, macrovascular disease is twice as common as microvascular disease, and fatal macrovascular disease is 70 times more common than fatal microvascular disease.$^{28} \mathrm{New}$ therapies are available or expected soon, including troglitazone and inhibitors of gluconeogenesis or lipolysis. ${ }^{29}$ Approval of these therapies by the FDA depends on the ability of the drugs to reduce levels of blood glucose or glycosylated hemoglobin in small, short-term trials even though these drugs are likely to be used by millions of dia-

JAMA, August 25, 1999-Vol 282, No. $8 \mathbf{7 8 7}$ 
betic patients for extended periods. The liver damage associated with troglitazone is an example of the postapproval discovery of major toxicity that led to the drug's being withdrawn in the United Kingdom ${ }^{30}$ and perhaps dampened enthusiasm for the therapy in the United States. ${ }^{31}$

\section{Validating a Surrogate as a Risk Factor vs Using a Surrogate to Evaluate a New Therapy}

The literature on surrogate end points often has failed to distinguish between the effort to validate a surrogate end point as a risk factor and the use of a surrogate end point to evaluate a new therapy. In the first instance, the validation of a surrogate end point is similar to showing that a risk factor is likely to be the cause of a disease. Strong, consistent, and independent demonstrations of the association in observational studies help to establish the factor as a "risk factor" for the outcome. Randomized trials showing that some form of treatment reduces both the level of the risk factor and the occurrence of the outcome of interest provide good evidence for a causal association. Unfortunately, randomized trials are not tests of particular risk factors or mechanisms of therapies. They are always trials of particular interventions, often specific drugs in particular doses. One trial of a drug from a single class does not provide much information about the validity of the surrogate end point because the evaluated therapy may in fact work through a mechanism completely independent of the surrogate. In other words, surrogate end points are best validated as "risk factors" by multiple trials using drugs from a variety of drug classes. With this sort of evidence, we can be more confident about our conclusion of the status of the "surrogate end point" as a "cause" of the disease.

In the second instance, a surrogate end point is used to evaluate a new therapy as safe and effective. This activity involves generalizing from one drug or class of drugs to another drug or class of drugs. The argument for the approval of a new drug on the basis of surrogates takes the following form: (1) in large, long-term clinical trials, drug A reduces both the surrogate end point and the disease incidence; (2) in short-term trials, drug B reduces the level of the surrogate end point; (3) in clinical practice, drug B will behave like drug A in its effect on disease incidence. In this argument by analogy, the net health effects of the untested drug are simply assumed to be the same as the net health effects of the tested drug. No empirical evidence exists to establish that these assumed benefits are not meaningfully offset by some combination of the known risks, the demonstrated adverse effects, or the unintended, unrecognized, and undocumented effects of the new drug.

The generalization from 1 tested therapy to a new therapy is most likely to be true when the 2 drugs are similar. If drugs $A$ and $B$ are from different drug classes, then their disparate mechanisms of action or adverse effects make the generalization from drug A's demonstrated benefit to drug B's hypothesized benefit more uncertain and tenuous. In other words, the use of a surrogate end point to evaluate a new therapy is most likely to be valid when the 2 therapies represent similar drugs, preferably from the same drug class. Paradoxically, surrogates are most likely valid where least needed. Of course, exceptions to the criteria for validating surrogates or evaluating therapies are possible. For example, the findings for high-dose and low-dose diuretic therapy on the outcome of coronary disease were not the same even though both therapies came from the same drug class. ${ }^{19}$

Because several antihypertensive drug classes, including lowdose diuretics and $\beta$-blockers, have been associated with a reduced risk of myocardial infarction, heart failure, or stroke, high blood pressure may be regarded as a validated risk factor for cardiovascular disease. The use of high blood pressure as a surrogate end point to evaluate a new therapy nonetheless remains problematic, especially for new drugs from new drug classes. Given the various actions of antihypertensive drugs-diuretics, $\beta$-blockers, calcium channel blockers, $\alpha$-blockers, and angiotensin-converting enzyme inhibitors-the assumption of equal net health effects across the drug classes seems questionable. Despite comparable effects on blood pressure and other risk factors, for example, longacting calcium channel blockers are associated with a higher risk of cardiovascular events than the angiotensinconverting enzyme inhibitors in hypertensive patients with type 2 diabetes mellitus. ${ }^{32-34}$ Even if all available classes of antihypertensive drugs had been tested and shown to reduce the incidence of cardiovascular disease in large, long-term trials, it remains an open question whether the health benefits associated with the tested drug classes would necessarily be generalizable to the health outcomes of the next new class of drugs that may lower blood pressure in short-term trials.

To focus on the surrogate end point as if we could intervene directly on the risk factor per se is to ignore major differences among pharmacologic agents. Conceptualizing the issue in terms of "the surrogate end point"-drug effects on risk factor levels-unfortunately encourages easy extrapolation from one drug to another drug even though the mechanisms of action, the adverse effects, and the effects on clinical end points may differ markedly between drugs or drug classes. For the fibrates, cholesterol lowering appears to be inadequate to counteract other drug effects that increase mortality. ${ }^{15-17}$ For the statins, the stabilization of plaques, ${ }^{35}$ the reduction in isoprenoids, ${ }^{36}$ and the effects on platelets, coagulation, endothelial function, and inflammatory responses $^{37,38}$ may be important mechanisms that provide health benefits independent of their ability to lower cholesterol.

\section{Proposed Revisions to the Drug-Approval Process}

Major revisions to the drug-approval process in the United States have been driven historically by drug tragedies. In 1938 , the FDA acquired new authority to regulate drug safety after about 107 children died after consuming a poisonous ingredient in a sulfonamide elixir. ${ }^{39}$ In 1962, the thalidomide tragedy renewed interest in drug regulation and led to the Kefauver-Harris Drug Amendments, which required 
that pharmaceutical companies, before marketing their drugs, demonstrate not only drug safety but also the effectiveness for intended use. ${ }^{39}$

The 1962 requirement for adequate and well-controlled studies is currently satisfied by surrogate end point trials for drug therapies of cardiovascular risk factors. Although surrogate end points are important early in the evaluation of drugs in development, their use in the drug-approval process for the treatment of what are generally asymptomatic risk factors does not provide an evaluation of meaningful clinical effectiveness and limits the ability to assess safety by permitting evaluation of the drug in fewer people exposed for a shorter time. In the late 1990s, millions of Americans are taking antihypertensive and antidiabetic therapies that have not been adequately evaluated in large, long-term clinical trials. Despite the widespread use of calcium channel blockers, angiotensin-converting enzyme inhibitors, $\alpha$-blockers, sulfonylureas, metformin, and troglitazone, their optimal role in the treatment of hypertension and type 2 diabetes mellitus based on clinical trials remains unclear. From the point of view of public health, drugs that may be used for many years by millions of people should be adequately evaluated..$^{40}$

As Califf and Kramer ${ }^{41}$ point out, "Large, randomized trials assessing therapies for common chronic diseases such as hypertension need to happen sooner rather than later in the development of new therapies, and these same types of trials are needed to identify the older therapies that are truly beneficial." They suggest that "physicians and health care providers take the lead in pointing out the need for adequate outcome data." ${ }^{41}$ While this strategy might work well in a few instances, relying on calls for clinical trials by clinicians is unlikely to provide a consistent and systematic approach to the evaluation of the safety and efficacy of drug therapies, old or new.

One systematic approach is a requirement that, prior to their approval, new drug therapies for cardiovascular risk factors should be evaluated in large, long-term clinical trials to assess their effects on major disease end points. The use of surrogate end points is avoided, and the major health outcomes are known prior to marketing. Such an approach would slow the time to drug approval and may meet with resistance from pharmaceutical manufacturers.

An alternative systematic approach is a requirement for the regular use of phase 4 trials in the approval of new drug therapies for cardiovascular risk factors. This strategy would represent an incremental improvement over the current approach, which usually requires little evaluation other than the use of surrogate end points in small, short-term studies. These required phase 4 studies should be large, long-term clinical trials designed to assess the effects of drug therapies on major disease end points over 3 to 5 years. The evaluation of the HMG-CoA reductase inhibitors is an excellent model. Initially approved on the basis of their ability to lower cholesterol in small, short-term studies, HMG-CoA reductase inhibitors have been associated with major reductions in the risk of heart disease and stroke in large, long-term tri- als. ${ }^{11,12,18}$ Unfortunately, we lack comparable information for most drug therapies for hypertension, diabetes, and obesity.

Even such a simple proposal as the regular requirement of large, long-term phase 4 trials raises a number of questions. This approach implicitly, though provisionally, accepts the validity of surrogate end points. Also, recruitment of subjects for a trial of an approved drug may be difficult. Does every new drug in a class require 1 large, longterm phase 4 trial for the evaluation of health outcomes? The time and costs of conducting health-outcome trials need to be balanced against the information they would yield. However, given the immense costs of long-term treatment of a large proportion of the population, it is difficult to justify cost as a reason not to conduct large, long-term trials of drug therapies for cardiovascular risk factors.

While the proposal to adopt phase 3 or phase 4 trials of health outcomes for new therapies of cardiovascular risk factors has drawbacks, the current system fails to encourage the clinical trials that are most important for clinical practice. ${ }^{42}$ In the late 20th century, the FDA drug-approval process may benefit from revisions that reflect both the advances and the limitations of our current understanding of drug therapies for cardiovascular risk factors.

Funding/Support: The research reported in this article was supported in part by grants HL40628, HL43201, and HL60739 from the National Heart, Lung, and Blood Institute; AG09556 from the National Institute on Aging, Bethesda, Md; and from the NWO (Nederlandse Organisatie voor Wetenschappelijk Onderzoek), The Hague, the Netherlands. Dr Psaty is a Merck/Society for Epidemiologic Research (SER) Clinical Epidemiology Fellow (cosponsored by the Merck Co Foundation, Rahway, NJ, and the SER, Baltimore, Md).

\section{REFERENCES}

1. Manson JE, Faich GA. Pharmacotherapy for obesity-do the benefits outweigh the risks [editorial]? N Engl J Med. 1996;335:659-660.

2. Abenhaim L, Moride $Y$, Brenot $F$, et al. Appetite-suppressant drugs and the risk of primary pulmonary hypertension. N Engl J Med. 1996;335:609-616.

3. Guy-Grand B, Apfelbaum M, Crepaldi G, Gries A, Lefebvre P, Turner P. International trial of long-term dexfenfluramine in obesity. Lancet. 1989;2:1142-1145.

4. Advisory committee votes on dexfenfluramine. Answers 11/27/1995. Available at: http://www.fda.gov/bbs/topics/ANSWERS/ANS00698.html. Accessed July 20, 1999.

5. Curfman GD. Diet pills redux [editorial]. N Engl J Med. 1997;337:629.

6. Connolly HM, Crary JL, McGoon MD, et al. Valvular heart disease associated with fenfluramine-phentermine. N Engl J Med. 1997;337:581-588.

7. McCann UD, Seiden LS, Rubin LJ, Ricaurte GA. Brain serotonin neurotoxicity and primary pulmonary hypertension from fenfluramine and dexfenfluramine. JAMA. 1997;278:666-672.

8. Fleming TR, DeMets DL. Surrogate end points in clinical trials: are we being misled? Ann Intern Med. 1996;125:605-613.

9. Psaty BM, Siscovick DS, Weiss NS, et al. Hypertension and outcomes research: from clinical trials to clinical epidemiology. Am J Hypertens. 1996;9:178-183.

10. SHEP Cooperative Research Group. Prevention of stroke by antihypertensive drug treatment in older persons with isolated systolic hypertension: final results of the Systolic Hypertension in the Elderly Program (SHEP). JAMA. 1991;265:3255-3264

11. Scandinavian Simvastatin Survival Study Group. Randomised trial of cholesterol lowering in 4444 patients with coronary heart disease: the Scandinavian Simvastatin Survival Study (4S). Lancet. 1994;344:1383-1389.

12. Shepherd J, Cobbe SM, Isles CG, et al, for the West of Scotland Coronary Prevention Study Group. Prevention of coronary heart disease with pravastatin in men with hypercholesterolemia. N Engl J Med. 1995;333:1301-1307.

13. Lipicky RJ, Packer M. Role of surrogate end points in the evaluation of drugs for heart failure. Am J Cardiol. 1993;22(suppl A):179A-184A.

14. Sobel BE, Furberg CD. Surrogates, semantics, and sensible public policy. Circulation. 1997;95:1661-1663.

15. Report from the Committee of Principal Investigators. A co-operative trial in the primary prevention of ischaemic heart disease using clofibrate. Br Heart J. 1978; 40:1069-1118. 
16. Oliver MF, Heady JA, Morris JN, Cooper J, for the Committee of Principal Investigators. WHO cooperative trial on primary prevention of ischaemic heart dis ease with clofibrate to lower serum cholesterol: final mortality follow-up. Lancet 1984;2:600-604

17. Gould AL, Rossouw JE, Santanello NC, Heyse JF, Furberg CD. Cholesterol reduction yields clinical benefit. Circulation. 1995;91:2274-2282

18. Downs JR, Clearfield $M$, Weis $S$, et al. Primary prevention of acute coronary events with lovastatin in men and women with average cholesterol levels: results of AFCAPS/TexCAPS. JAMA. 1998:279:1615-1622.

19. Psaty BM, Smith NS, Siscovick DS, et al. Health outcomes associated with antihypertensive therapies used as first-line agents: a systematic review and metaanalysis. JAMA. 1997;277:739-745.

20. Temple RJ. A regulatory authority's opinion about surrogate endpoints. In: Nimmo WS, Tucker GT, eds. Clinical Measurement in Drug Evaluation. New York NY: John Wiley \& Sons; 1995:3-22.

21. Meinert $C L$, Knatterud $G L$, Proust $T E$, Klimt CR. University Group Diabetes Program, II: mortality results. Diabetes. 1970;19(suppl I):789-830.

22. The Diabetes Control and Complications Trial Research Group. The effect of intensive treatment of diabetes on the development and progression of longterm complications in insulin-dependent diabetes mellitus. N Engl J Med. 1993; 329:977-986.

23. Ohkubo $Y$, Kishikawa $H$, Araki $E$, et al. Intensive insulin therapy prevents the progression of diabetic microvascular complications in Japanese patients with noninsulin-dependent diabetes mellitus: a randomized prospective 6-year study. Diabetes Res Clin Pract. 1995;28:103-117.

24. Abraira C, Colwell J, Nuttall F, et al. Cardiovascular events and correlates in the Veterans Affairs Diabetes Feasibility Trial. Arch Intern Med. 1997:157:181-188. 25. UK Prospective Diabetes Study (UKPDS) Group. Intensive blood-glucose con trol with sulphonylureas or insulin compared with conventional treatment and risk of complications in patients with type 2 diabetes (UKPDS 33). Lancet. 1998;352: 837-853.

26. Nathan DM. Some answers, more controversy, from UKPDS [commentary] Lancet. 1998;352:832-833.

27. Barrett-Connor E. Does hyperglycemia really cause coronary heart disease? Diabetes Care. 1997;20:1620-1623.
28. Turner R, Cull C, Holman R. United Kingdom Prospective Diabetes Study 17: a 9-year update of a randomized, controlled trial on the effect of improved metabolic control on complications in non-insulin-dependent diabetes mellitus. Ann Intern Med. 1996;124:136-145.

29. Dagogo-Jack S, Santiago JV Pathophysiology of type 2 diabetes and modes of action of therapueutic interventions. Arch Intern Med. 1997;157:1802-1817. 30. Mitchell P. Shock as troglitazone withdrawn in UK. Lancet. 1997;350:1685. 31. Imura H. A novel antidiabetic drug, troglitazone-reason for hope and concern [editorial]. N Eng/ J Med. 1998;338:908-909.

32. Estacio RO, Jeffers BW, Hiatt MR, et al. The effect of nisolidipine as compared with enalapril on cardiovascular outcomes in patients with non-insulindependent diabetes and hypertension. N Engl J Med. 1998;338:645-652.

33. Tatti $P$, Pahor M, Byington RP, et al. Outcome results of the Fosinopril versus Amlodipine Cardiovascular Events Trial (FACET) in patients with hypertension and non-insulin dependent diabetes mellitus. Diabetes Care. 1998;21:597-603.

34. Pahor M, Psaty BM, Furberg CD. Treatment of hypertensive patients with diabetes [commentary]. Lancet. 1998;351:689-690.

35. Brown BG, Zhao X, Sacco DE, Albers JJ. Lipid lowering and plaque regression: new insights into prevention of plaque disruption and clinical events in coronary disease. Circulation. 1993:87:1781-1791.

36. Massy ZA, Keane WF, Kasiske B. Inhibition of the mevalonate pathway: benefits beyond cholesterol reduction? Lancet. 1996;347:102-103.

37. Vaughn CJ, Murphy MB, Buckley BM. Statins do more than just lower cholesterol. Lancet. 1996;348:1079-1082.

38. Rosenson RS, Tangney CC. Antiatherothrombotic properties of statins: implications for cardiovascular event reduction. JAMA. 1998;279:1643-1650.

39. Food and Drug Administration. The evolution of U.S. drug law. Available at: http://www.fda.gov/fdac/special/newdrug/benlaw.html. Accessed July 1999.

40. Ferner RE. Newly licensed drugs should be on probation until their value is demonstated. BMJ. 1996:313:1157-1158.

41. Califf RM, Kramer JM. What have we learned from the calcium channel blocker controversy [editorial]? Circulation. 1998;97:1529-1531.

42. Davis BR, Cutler JA, Gordon DJ, et al, for the ALLHAT Research Group. Rationale and design for the Antihypertensive and Lipid Lowering Treatment to Prevent Heart Attack Trial (ALLHAT). Am J Hypertens. 1996;9:342-360.

\section{Are Surrogate Markers Adequate to Assess Cardiovascular Disease Drugs?}

Robert Temple, MD

$\mathrm{T}$

HE USE OF SURROGATE END POINTS AS A BASIS FOR REACHing conclusions about the benefit of therapy has been met with both rising enthusiasm, reflected in recent changes in the Food, Drug and Cosmetic Act ${ }^{1}$ and some recent US Food and Drug Administration (FDA) actions, and rising concern, reflected in several strongly stated warnings..$^{2-4}$ The mixed response is not surprising: reliance on surrogates, when the surrogate proves to predict clinical benefit, can bring treatment benefits to patients years before information on clinical outcomes could be available and at relatively low cost. But reliance on surrogates, when the effect on the surrogate does not lead to clinical benefit, can lead to the adoption of useless or even harmful therapies. The obvious community goal is to make decisions most likely to yield the former of these outcomes.

\section{Defining Surrogate End Points}

As defined in the preamble to the FDA's proposed accelerated approval rule, "A surrogate end point, or 'marker,' is a

\section{See also p 786.}

laboratory measurement or physical sign that is used in therapeutic trials as a substitute for a clinically meaningful end point that is a direct measure of how a patient feels, functions, or survives and is expected to predict the effect of the therapy." An effect on the surrogate end point is thus not per se of any value to the patient. It is a benefit only to the extent that it causes or predicts an improved outcome (fewer myocardial infarctions, strokes, or deaths). Most surrogates are chosen because they are thought to be on the causal chain leading to the clinical outcome. Surrogates can be early or late in the presumed causal chain: cholesterol (a biochemical variable), blood pressure (a pathophysiologic variable), and coronary vessel diameter and left ventricular hypertrophy (morphological variables) are all surrogates, ${ }^{4}$ but the last 2 are closer to certain clinical events (myocardial infarction and heart failure). Some surrogates are not etiologic but are thought to reflect the activity of the underlying process that leads to adverse outcomes. Some surrogates can be thought of qualitatively (the

Author Affiliation: Center for Drug Evaluation and Research, Food and Drug Administration, Rockville, Md.

Corresponding Author and Reprints: Robert Temple, MD, Center for Drug Evaluation and Research Policy, Food and Drug Administration, 5600 Fishers Ln, HFD101, Rockville, MD 20857 (e-mail: Temple@CDER.fda.gov).

Controversies Section Editor: Phil B. Fontanarosa, MD, Interim Coeditor 